\title{
Grandezze e misure fra aula e palestra
}

\section{Magnitudes and measures between the classroom and the school gym}

\author{
Luca Crivelli
}

Dipartimento formazione e apprendimento - SUPSI, Locarno, Svizzera

Sunto / Il presente contributo ha lo scopo di descrivere un percorso didattico rivolto agli allievi di scuola elementare, all'inizio del secondo ciclo', proposto e sperimentato sull'arco di un intero anno scolastico. Gli allievi sono confrontati con una situazione complessa: l'organizzazione di una giornata sportiva all'interno dell'istituto scolastico, chiamata Giochi Senza Frontiere delle Misure. Gli studenti lavorano dapprima sulle unità di misura convenzionali, ne indagano il funzionamento e imparano ad usare in modo appropriato gli strumenti di misura convenzionali; infine, viene richiesto loro di concepire e gestire alcuni giochi di squadra per i loro compagni di scuola. Le tematiche e gli argomenti trattati in aula nell'ambito Grandezze e misure diventano così dei giochi di movimento e di squadra in palestra, in un'ottica di interdisciplinarità fra matematica e motricità.

Parole chiave: situazione - problema; motricità; grandezze; misure.

\begin{abstract}
This contribution aims to present a didactic experience designed for primary school. The project, conducted in a third grade classroom throughout an entire school year, implied the organization of a sport tournament inspired by a popular TV Show called Giochi Senza Frontiere (It's a Knockout!). The students work on conventional units of measurement, investigate how they work, and also how to use instruments of measurement in an appropriate way; finally, they are asked to conceive and to handle some team games for their schoolmates. The topics developed in the classroom within the context Magnitudes and measures thus become games of movement and teamwork in the school gym, from an interdisciplinary perspective between mathematics and motor skills.
\end{abstract}

Keywords: realistic situation; sport; magnitudes; measure.

\section{1 introdurione}

I Giochi senza frontiere erano una manifestazione ludica e sportiva particolarmente conosciuta e apprezzata in Europa negli anni ' 80 e ' 90 del secolo scorso. Le squadre, provenienti da diversi paesi, si sfidavano in giochi di abilità, fortuna e resistenza, guadagnando i punti necessari per vincere il torneo. Le varie prove, bizzarre e divertenti, erano caratterizzate da un tema comune, spesso strettamente legato alla nazione che ospitava la competizione.

L'idea di sperimentare un percorso ispirato a questi giochi, ormai divenuti leggendari per chi ha avuto la fortuna di seguirli in televisione, è nata dalla volontà di proporre delle attività che portassero la matematica fuori dall'aula scolastica, in particolare negli spazi esterni alla scuola e dentro alla palestra. Per la progettazione delle attività del percorso si è presa ispirazione da alcuni materiali e libri in circolazione (Brandenberg et al., 2013; Cottino et al., 2011; Daynes, 2016; Novelli, 2016), riformulati, adattati e integrati con materiali ideati dai docenti.

1. In Canton Ticino, I'inizio del secondo ciclo coincide con la classe terza elementare. 
La classe per cui è pensato il progetto è una terza elementare. Gli sperimentatori sono due docenti titolari di scuola elementare, Luca Crivelli e Vanessa Henauer, in collaborazione con la docente di educazione fisica Simona Cantù.

L'interdisciplinarietà non è l'unico focus: le varie fasi del percorso sono caratterizzate da un'attenzione particolare nei confronti della valutazione. Come descritto in particolare nel par. 4, per monitorare l'evoluzione delle competenze degli allievi si sceglie di favorire una pluralità di sguardi (valutazione da parte del docente, dei compagni, dell'allievo stesso) e di strumenti, con I'obiettivo di creare una rubrica valutativa che funga da raccoglitore per tutte le informazioni rilevate. Tale rubrica permette di tracciare un quadro completo delle competenze degli allievi nell'arco dell'intero anno scolastico.

\section{Quadro organizzativo}

Il percorso prevede una grande situazione - problema ${ }^{2}$ da affrontare e risolvere: gli allievi sono chiamati a sfruttare le competenze costruite durante l'intero anno scolastico, nell'ambito disciplinare di Grandezze e misure (DECS, 2015), per progettare e proporre al resto dell'istituto scolastico delle sfide ludiche e sportive. II percorso è articolato in cinque fasi distinte: la prima prevede che la situazione problema venga lanciata e condivisa con la classe. La seconda, la terza e la quarta fase sono legate all'approfondimento di alcune grandezze e misure (misure di tempo, misure di lunghezza e misure di massa e capacità). II progetto si conclude con l'ideazione e la realizzazione delle sfide sportive che sono proposte nella giornata di Giochi Senza Frontiere: in quest'ultima fase, gli allievi devono implicarsi e mettere a frutto quanto allenato e visto in classe, effettuando misurazioni legate alle diverse grandezze e confrontando i numeri ottenuti per stabilire i vincitori.

Per avere un'idea di come l'intero percorso è distribuito sull'arco dell'anno scolastico, da settembre a giugno, si osservi la Figura 1.

Figura 1

Rappresentazione grafica della temporalità del percorso, da settembre a giugno.

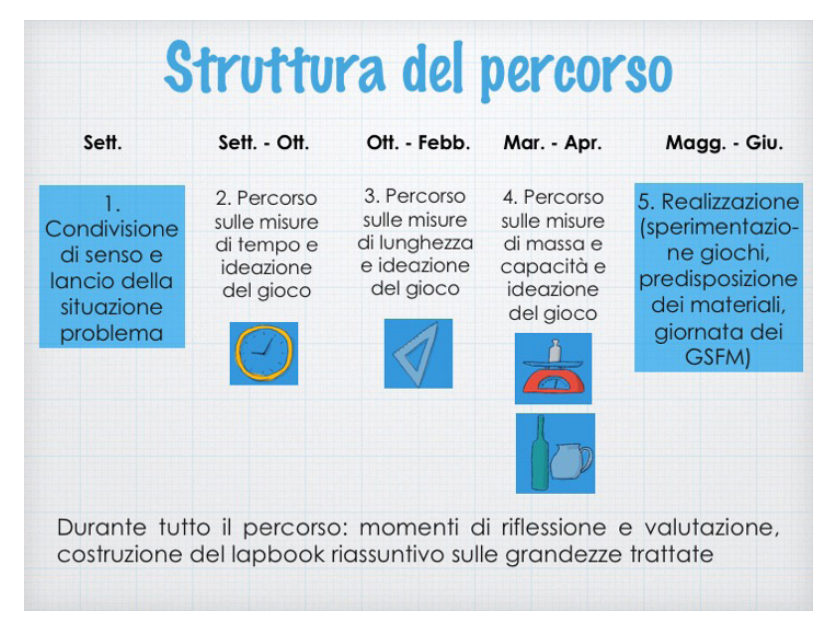

2. Secondo Castoldi (2011, p. 186), la situazione-problema è «un problema da risolvere in un dato contesto operativo, all'interno dei vincoli e delle risorse poste dal contesto stesso», ovvero l'oggetto stesso, il fine ultimo del progetto. 
Prevedendo un tempo di lavoro di 1-2 unità didattiche ${ }^{3}$ settimanali, il percorso si

svolge durante l'intero anno scolastico, per un totale di circa 35 momenti, e si può quindi considerare parte integrante della progettazione annuale di matematica per l'ambito Grandezze e misure. Molte delle attività inserite nel progetto sono a carattere interdisciplinare e possono trovare spazio, nella griglia oraria settimanale, durante le ore previste per educazione fisica o per arti plastiche.

\section{Il percorso didattico}

Trattandosi di un percorso lungo e complesso, l'articolazione operativa descritta di seguito è stata suddivisa in più sottoparagrafi, in modo da rendere la descrizione delle attività più fruibile e meno dispersiva. Vista la mole di contenuti, è stato necessario un importante lavoro di sintesi: in allegato, è possibile trovare i materiali concreti utilizzati in classe o citati solo superficialmente nel testo, così da farsi un'idea più precisa dell'effettivo lavoro svolto in classe, e poter eventualmente replicare alcuni dei momenti d'apprendimento descritti.

\subsection{Lancio della situazione - problema}

Prima di lanciare la situazione - problema della giornata sportiva, viene proposta un'attività introduttiva sulle grandezze, che ha la funzione di far chiarezza sulla terminologia che poi verrà impiegata durante il resto del percorso. II docente mostra alla classe diversi oggetti (un orologio, una bottiglia, una statuetta, un filo, una clessidra, una mela) e chiede ai ragazzi di trovare delle singole parole che possano descriverli. In seguito, chiede loro di selezionare, fra tutte le qualità scritte alla lavagna, solo quelle che si possono misurare; ad esempio, non si può attribuire un valore numerico al colore di un oggetto, mentre è possibile farlo per la sua lunghezza, o per la sua massa. Al termine della discussione viene fatta chiarezza su alcuni termini e concetti: gli oggetti che ci circondano hanno delle qualità. Alcune di queste qualità si possono misurare: si tratta delle grandezze. Misurare significa attribuire, tramite uno strumento e rifacendosi a un'unità di misura, un valore alla grandezza in questione (Figura 2).

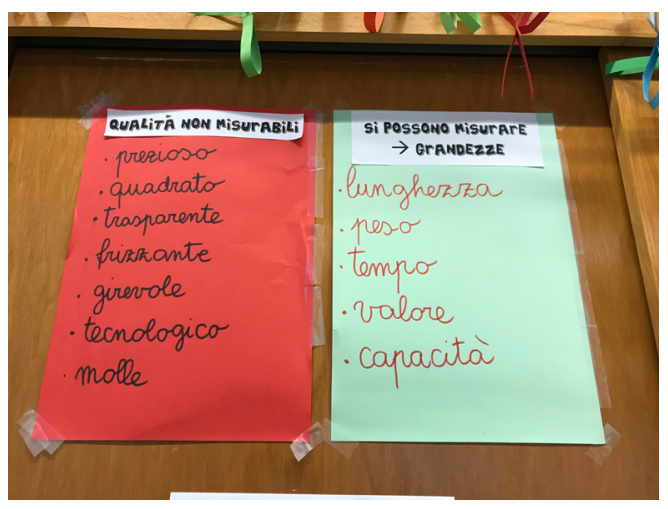


Alla classe è quindi proposto un filmato risalente agli anni '90, un estratto dei Giochi Senza Frontiere (Link). Al termine del filmato, il docente pone alcune domande atte a verificare che la classe abbia compreso la natura e lo spirito dei giochi: cosa rappresentano le diverse squadre? Qual è il tema che accomuna le varie sfide? Come si stabilisce il vincitore? Quale tipo di gioco viene proposto?

La situazione - problema viene quindi lanciata: in collaborazione con la docente di educazione fisica, la classe si occuperà di organizzare una grande giornata di Giochi Senza Frontiere delle Misure (in seguito GSFM). Viene avviata una prima discussione, in cui il docente esplicita alcuni elementi essenziali volti a facilitarne l'organizzazione:

- i GSFM saranno la giornata di chiusura dell'anno scolastico, in giugno;

- parteciperanno tutti gli allievi dell'istituto, dalla scuola dell'infanzia alla quinta elementare;

- i giochi avranno un tema in comune, cioè quello delle grandezze e delle rispettive misure;

- i giochi verranno organizzati nel corso dell'anno, in seguito a percorsi più o meno brevi legati alle misure di tempo, lunghezza, massa e capacità (descritti sinteticamente nei par. 3.2, 3.3 e 3.4);

- la classe terza, cioè quella direttamente coinvolta nella sperimentazione, sarà responsabile dello svolgimento dell'intera giornata.

Per tenere una traccia visibile durante l'intero percorso, il docente propone la realizzazione di un lapbook legato alle grandezze e alle misure (Figura 3). II lapbook può essere definito come un particolare tipo di cartellone riassuntivo e interattivo: ci sono linguette, parti mobili, carte estraibili e piccoli testi (Gottardi \& Gottardi, 2016). Inizialmente il lapbook ha solo il titolo, ma al termine dell'anno sarà completo con informazioni e promemoria di ogni tipo.

Lo strumento è pensato per essere personalizzato con annotazioni degli allievi, e avrà una doppia funzione: da un lato, potrà essere utilizzato come mezzo per istituzionalizzare e fissare alcuni apprendimenti e scoperte fatte in classe; dall'altro, riguardandone gli elementi, sarà possibile allenarsi e ritornare sui numerosi concetti messi in evidenza nelle diverse fasi del percorso. II lapbook scelto per questo particolare percorso è una rielaborazione di quello progettato e pubblicato da Gottardi e Gottardi (2016).

Figura 3

Il lapbook completato,

fotografato alla fine

dell'anno scolastico.
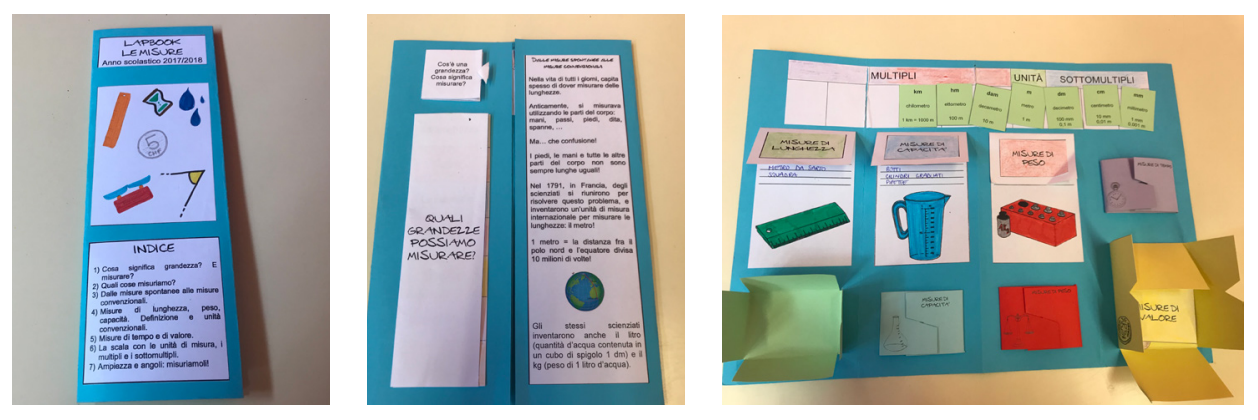

\subsection{Le misure di tempo e la lettura dell'orologio}

La prima fase del percorso prevede un approfondimento sulle misure di tempo, con particolare attenzione alla lettura dell'orologio analogico. Prima ancora di iniziare, il docente chiede alla classe di portare da casa degli strumenti che possano essere utilizzati per misurare il tempo. Le prime attività proposte hanno il semplice scopo 
di far vivere agli allievi esperienze significative che prevedano l'utilizzo spontaneo di tali strumenti: un esempio su tutti sono delle semplici sfide di abilità, in cui ognuno possa registrare il tempo impiegato per compiere determinate azioni: quanto tempo si impiega a costruire una torre di cubi di legno? Quante volte, in un minuto, si riesce a scrivere il proprio nome su di un foglio bianco?

Giunge poi una nuova richiesta da parte del docente: oltre agli strumenti portati da casa, in classe sono da sempre a disposizione delle clessidre. Purtroppo, però, hanno tutte una durata standard di un minuto, e a volte non sono comode per misurare altri intervalli di tempo: sarebbe interessante avere uno strumento, per esempio, che possa essere impiegato per misurare il tempo a disposizione di ogni allievo quando, al lunedì mattina, vuole raccontare il proprio fine settimana ai compagni. Viene così lanciata un'attività laboratoriale che riguarda la costruzione di clessidre, utilizzando materiale di fortuna recuperato a casa o nell'aula. A gruppi, gli allievi stabiliscono una durata indicativa della clessidra che intendono costruire, e realizzano dapprima un progetto, illustrandone le parti importanti e il funzionamento. In un secondo tempo, si passa al recupero del materiale e alla costruzione vera e propria dello strumento. Al termine della costruzione, la durata di ogni clessidra viene misurata con degli orologi. II dato rilevato viene scritto su di un'etichetta, in seguito posta sulla clessidra stessa. Le clessidre costruite (Figura 4) rimangono a disposizione della classe e vengono utilizzate come timer, per esempio per stabilire i turni di parola in una discussione, o il tempo massimo da impiegare per riordinare l'aula al termine di un'attività.

Figura 4

Le clessidre costruite

durante l'attività

laboratoriale.

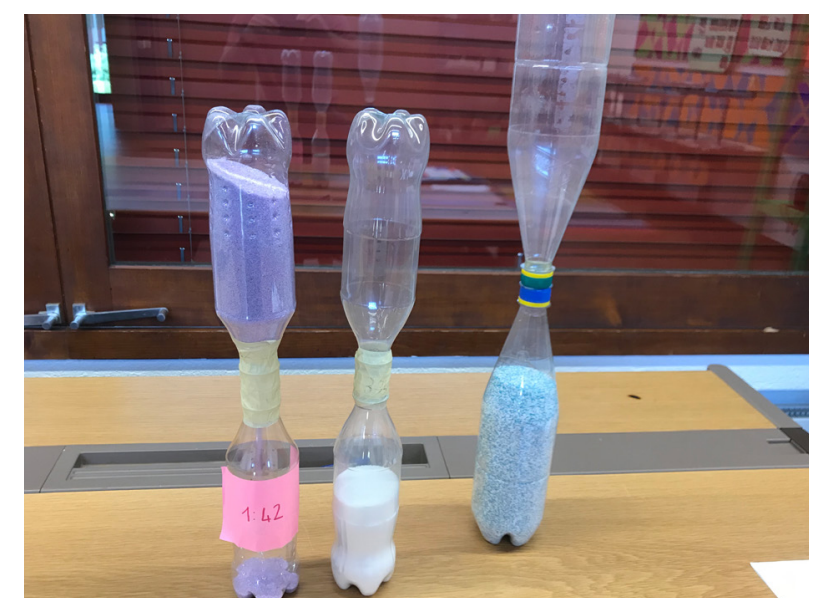

Viene quindi portata l'attenzione della classe su di un altro strumento che, almeno all'apparenza, è piuttosto conosciuto: I'orologio. Questa attività può essere introdotta proponendo il taboo matematico (Allegato 1); il gioco consiste nel far indovinare ai propri compagni, attraverso una descrizione verbale, strumenti di misura e termini legati alla matematica, con però il divieto di utilizzare alcune parole proibite indicate sulle carte. Se lo strumento da indovinare fosse proprio I'orologio, quali sarebbero le parole proibite che renderebbero difficile il gioco? La costruzione della carta del taboo orologio, fatta insieme agli allievi, diventa uno spunto per far emergere le loro conoscenze riguardanti questo strumento.

A partire dall'osservazione degli esempi portati da casa, il docente media una discussione volta a far chiarezza sui nomi delle parti che compongono l'orologio: in quelli analogici si può individuare il quadrante e le lancette di secondi, minuti e ore. 
In questa fase, si mette l'accento sulle differenze anche macroscopiche che ci sono fra i vari strumenti, facendo una distinzione fra orologio digitale, più facile da leggere, e quello analogico, leggermente più complesso. A coloro che sanno già leggere I'ora sull'orologio analogico viene chiesto di descrivere agli altri alcune tecniche e strategie efficaci. È in questo frangente che è utile proporre la progettazione e la realizzazione di un orologio didattico, uno strumento che l'allievo potrà in seguito utilizzare per allenarsi nel leggere o riprodurre degli orari (Figura 5). Ognuno riceve un quadrante, che andrà completato aggiungendo lancette e decorazioni a piacere. Il quadrante ha ben indicati i numeri delle ore, e delle piccole tacche che facilitano il conteggio dei minuti. Allo stesso tempo, sul quadrante è presente la distinzione fra le ore mattutine (dall'1 al 12) e quelle pomeridiane (dalle 13 alle 24); qualora non fosse ancora emerso il discorso, è l'occasione per affrontare, tramite discussione, questo aspetto particolare spesso meno conosciuto dai bambini.

Figura 5

Esempi di orologi didattici costruiti dagli allievi.

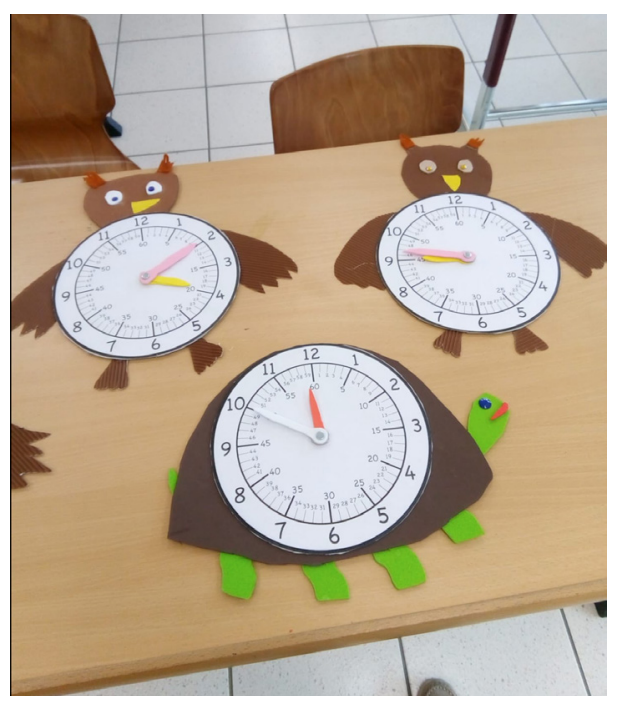

A questo punto, è utile proporre delle attività di allenamento in cui gli allievi meno competenti nella lettura dell'ora possano familiarizzare con essa giocando, e vivendo situazioni realistiche e concrete. Gli allievi più competenti già in partenza, invece, rivestono il ruolo di tutor, oppure possono dedicarsi all'utilizzo di un linguaggio sempre più specifico («meno un quarto», «e tre quarti» ecc.). Risulta interessante, in questo senso, la possibilità di proporre attività a postazioni, in cui esercitarsi nella lettura dell'orologio, e più in generale sul concetto di orario e di tempo che passa, in maniera differenziata:

1. Lancia e gira: si gioca in tre. II primo allievo lancia dei dadi, su cui sono indicate delle ore e dei minuti. II secondo allievo, dopo aver osservato i dadi, gira le lancette del proprio orologio o di un orologio didattico fornito dal docente, indicando l'orario ottenuto. II terzo allievo controlla che le lancette siano posizionate correttamente e descrive agli altri un'attività che è possibile fare a quell'ora del giorno. Si procede cambiando i ruoli (Figura 6).

2. Caccia alle parole: si tratta del classico gioco delle categorie, in cui per diverse categorie prestabilite (nomi, frutti ecc.) occorre trovare e scrivere delle parole che abbiano la stessa lettera iniziale. Sono gli allievi stessi a tenere il tempo, utilizzando un timer da cucina, e fermandosi quando questo scade (Allegato 2). 
3. Cosa? Quando?: ognuno riceve un pennarello e quattro carte, su cui sono indicate alcune possibili attività legate al quotidiano (prendere l'autobus, fare colazione, guardare la tv ecc.). Ogni allievo osserva le immagini, e prova ad assegnare a ognuna di esse un orario adeguato. Su ogni carta è indicato un orologio digitale e uno analogico, che sono da completare con l'orario scelto, utilizzando il pennarello. Quando tutti hanno completato le carte, le si riordina mettendole in ordine cronologico, dall'attività che si svolge più presto a quella che si svolge più tardi nell'arco della giornata.

Figura 6

Delle allieve giocano a riprodurre degli orari su di un orologio didattico, durante un'attività a postazioni.

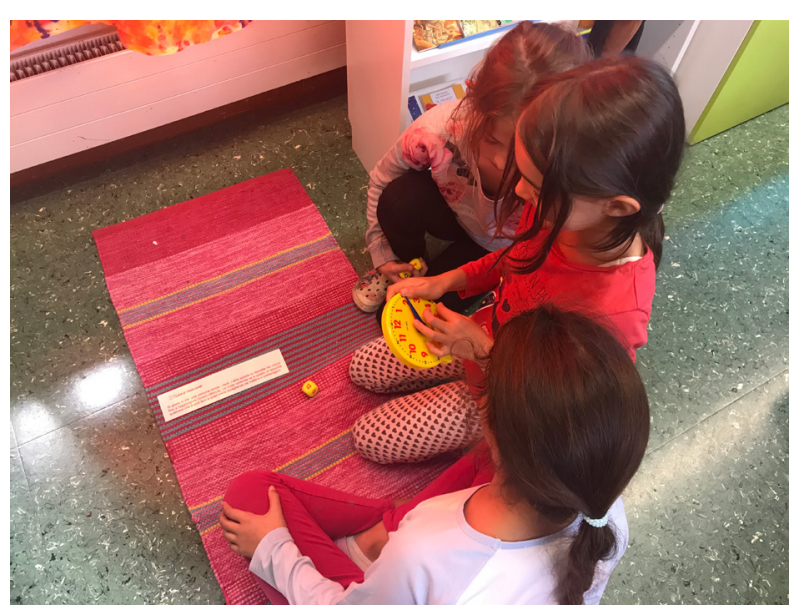

Come esplicitato in fase d'introduzione, I'interdisciplinarità con l'educazione fisica è uno degli aspetti focali attorno al quale è costruita l'intera progettazione. Per questo motivo, in questa fase del percorso sono previste anche attività di allenamento in palestra, come quella dell'orologio vivente. II gioco prevede la collaborazione da parte di tutti gli allievi: due bambini dirigono i lavori, mentre gli altri eseguono ciò che viene loro richiesto dai compagni. Parte della classe forma, con i propri corpi e sdraiandosi a terra, il quadrante dell'orologio. II lavoro consiste nel rispondere alle domande di un quiz. La soluzione alle domande poste corrisponde a un orario (ad esempio, "A che ora finisce la scuola al pomeriggio?»). I due allievi che dirigono l'attività devono far sdraiare due compagni, che rappresentano le lancette, in modo tale che l'orario rappresentato dall'orologio umano sia la risposta alla domanda del quiz posta dal docente (Figura 7).

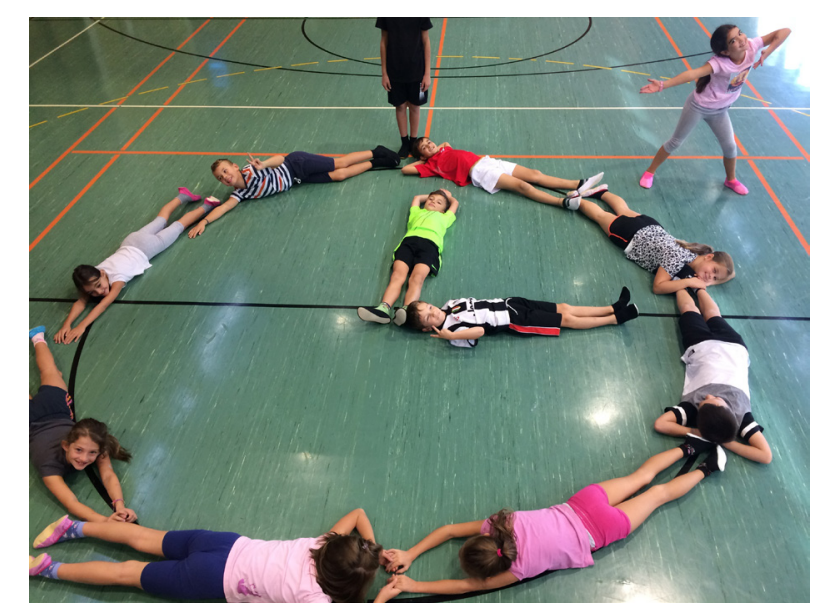

\section{Figura 7}

Parte della classe riproduce un orario, le 15:00 in punto, sull'orologio vivente. 
Sempre in collaborazione con il docente di educazione fisica, è possibile invitare gli allievi a utilizzare strumenti di misurazione come orologi e cronometri anche durante le normali lezioni in palestra. Allo stesso tempo, può essere interessante proporre discussioni legate al tempo e allo sport: in quali discipline le prestazioni degli atleti dipendono dal tempo impiegato per svolgere una determinata azione?

\subsection{Le misure di lunghezza}

Si tratta della fase del progetto più lunga e articolata, perché riguarda principalmente la scoperta della necessità e dell'utilità delle unità di misura convenzionali, fatta tramite attività di esplorazione ma anche ripercorrendo alcune tappe della storia della matematica relative a questa tematica.

Il percorso sulle misure di lunghezza viene introdotto leggendo e mostrando le immagini delle prime pagine di un libretto intitolato Crictor, il serpente dal cuor d'oro (Ungerer, 1963). La lettura viene interrotta nel momento in cui la protagonista, Luisa, decide di confezionare un abitino di lana per il proprio serpente domestico. Si pone il quesito ai bambini: quanto lungo dovrà essere l'abito su misura che la Signora Luisa dovrà creare per il suo serpente? Da un cestino posto al centro dell'aula si fanno fuoriuscire dei serpenti di corda. I bambini suddivisi in gruppetti misurano la lunghezza del serpente-corda utilizzando parti del proprio corpo o degli oggetti che trovano in aula, e registrano i risultati ottenuti su una scheda accompagnatoria (Allegato 3). La misurazione viene effettuata dal gruppo quattro volte, cambiando sempre l'oggetto scelto come unità di riferimento. A fine lavoro viene lasciato un momento di spazio agli allievi per confrontarsi e mettere in comune impressioni, eventuali difficoltà o incertezze avute durante l'attività.

A distanza di qualche giorno, si propone quindi una nuova misurazione del serpente. Questa volta la scelta dell'unità di misura non sarà libera, ma sarà una parte del corpo o un oggetto non convenzionale particolare, scelta durante l'attività precedente da ciascun gruppo. I bambini, seguendo le indicazioni sull'oggetto da utilizzare, misurano la lunghezza del serpente-corda e indicano i risultati in una tabella (Allegato 4). Questa proposta permette il confronto tra le misurazioni rilevate, visto che gli oggetti utilizzati sono gli stessi per tutti.

Successivamente vengono presentati i dati registrati da ciascun gruppo. L'obiettivo è quello di mettere in evidenza le probabili differenze che a questo punto possono emergere. Perché un gruppo misurando la lunghezza del serpente con la ciabatta ha ottenuto un numero diverso dagli altri? II docente media una discussione sulle possibili motivazioni che stanno alla base della diversità dei risultati ottenuti. Dalla riflessione degli allievi sulle possibili cause (strumento uguale ma utilizzato in modo diverso, oggetto non perfettamente identico ecc.) dovrebbe emergere che è più opportuno scegliere alcuni strumenti già conosciuti, anche se non ancora molto utilizzati in classe, come il metro. D'altra parte, le problematiche nel trovare uno strumento comune che permetta di minimizzare le differenze tra i risultati delle misurazioni effettuate sono le stesse che hanno accompagnato l'uomo nel corso della storia. Per questo motivo, viene presentato un riassunto dell'evoluzione delle misure di lunghezza e dei vari metodi utilizzati nel corso degli anni, al fine di capire come e perché si sia giunti ad una soluzione condivisa dalla maggior parte dei paesi del mondo. Questa presentazione in Power Point (Allegato 5) ha lo scopo di fare chiarezza e di precisare l'importanza di avere uno strumento di misura convenzionale, di chiarire come si sia arrivati a stabilirlo e di come debba essere utilizzato.

Per approfondire l'argomento e per scoprire come sono costruite le unità di misura 
convenzionali per la lunghezza, vengono quindi proposte due attività laboratoriali distinte che permettono di lavorare con diversi strumenti e unità di misura (nello specifico metro, decimetro, centimetro e millimetro).

La prima proposta permette di comprendere e manipolare al meglio il metro come strumento e come unità di misura. I bambini, dopo aver sperimentato gli strumenti di misura messi a loro disposizione (metro da muratore, metro da sarto, riga da $1 \mathrm{~m}$ della lavagna, riga centimetrata da $50 \mathrm{~cm}$, riga da $30 \mathrm{~cm}$, righello da $15 \mathrm{~cm}$ ) hanno il compito di costruire un metro di cartoncino sul quale indicano, con colori differenti, le diverse unità di misura: il metro, il decimetro, il centimetro e il millimetro (Figura 8). Durante il lavoro è importante osservare e ascoltare i ragionamenti che i bambini esplicitano per poter rilanciare concetti o formulazioni corrette. Si potrebbero per esempio accorgere che $1 \mathrm{~m}$ e $35 \mathrm{~cm}$ equivalgono a $135 \mathrm{~cm}$, oppure che occorrono $10 \mathrm{dm}$ per ottenere $1 \mathrm{~m}$.

Figura 8

Esempio di metri in

cartoncino su cui sono indicate diverse unità di misura.
Figura 9

Esempio di linea del tempo appesa in classe.

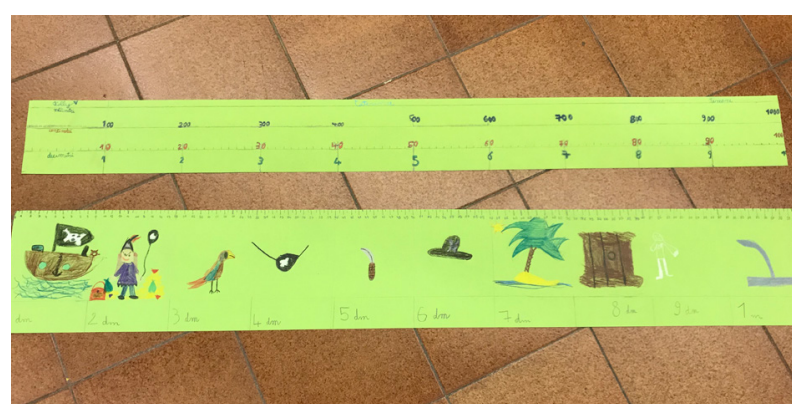

La seconda attività laboratoriale proposta riguarda la costruzione di una linea del tempo che indichi gli anni dal 1750 in poi. La linea del tempo, che in seguito verrà utilizzata durante le lezioni di geo-storia, deve avere alcune caratteristiche precise stabilite dal docente: per poter essere appesa alla parete dell'aula deve essere lunga al massimo 7 metri, deve indicare con precisione gli anni 1750, 1800, 1850, 1900, 1950, 2000 e 2018, e deve risultare facilmente leggibile una volta appesa sul muro in classe (Figura 9). Per posizionare al meglio i singoli anni e i particolari avvenimenti storici studiati in classe, gli allievi utilizzano il metro da muratore o da sarto. Il docente osserva, cercando di intervenire il meno possibile, e lasciando che i gruppi portino avanti in autonomia il proprio progetto. Anche in questa attività, gli allievi manipolano lo strumento convenzionale e ne approfondiscono il funzionamento.

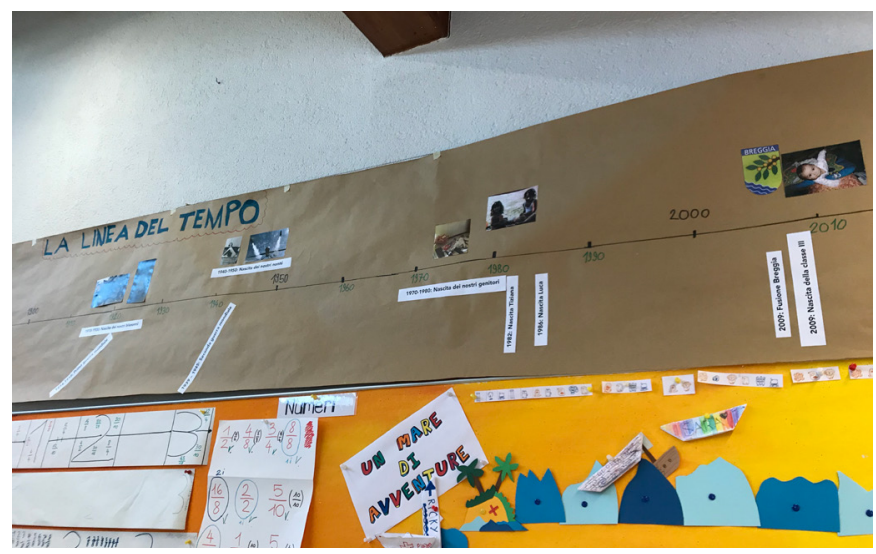


Per dare a tutti la possibilità di allenare sia l'utilizzo di uno strumento di misura convenzionale (la riga), sia la lettura di una misurazione, vengono quindi proposte quattro attività a postazioni:

- La gara di corsa: i bambini a turno, lanciano il dado, e avanzano lungo un percorso, rappresentato da una linea spezzata (Allegato 6), di un numero di centimetri pari al numero ottenuto con il dado. Per misurare la distanza da percorrere utilizzano la riga centimetrata. Ogni volta segnano il punto raggiunto con un pennarello, e passano il turno al compagno. Vince chi arriva per primo al traguardo.

- Dadi e stima: a turno, i bambini lanciano due dadi (uno su cui sono scritte le sigle $\mathrm{mm}, \mathrm{cm}, \mathrm{dm}$ e $\mathrm{m}$, e un altro con i numeri da 1 a 10). Chi ha lanciato il dado pensa a un oggetto che possa essere di quella lunghezza. Se i compagni sono d'accordo, il bambino ottiene 1 punto. Se chi ha lanciato il dado riesce a prendere I'oggetto e a portarlo agli altri, ottiene 2 punti. Vince chi ha più punti alla fine della partita (Figura 10).

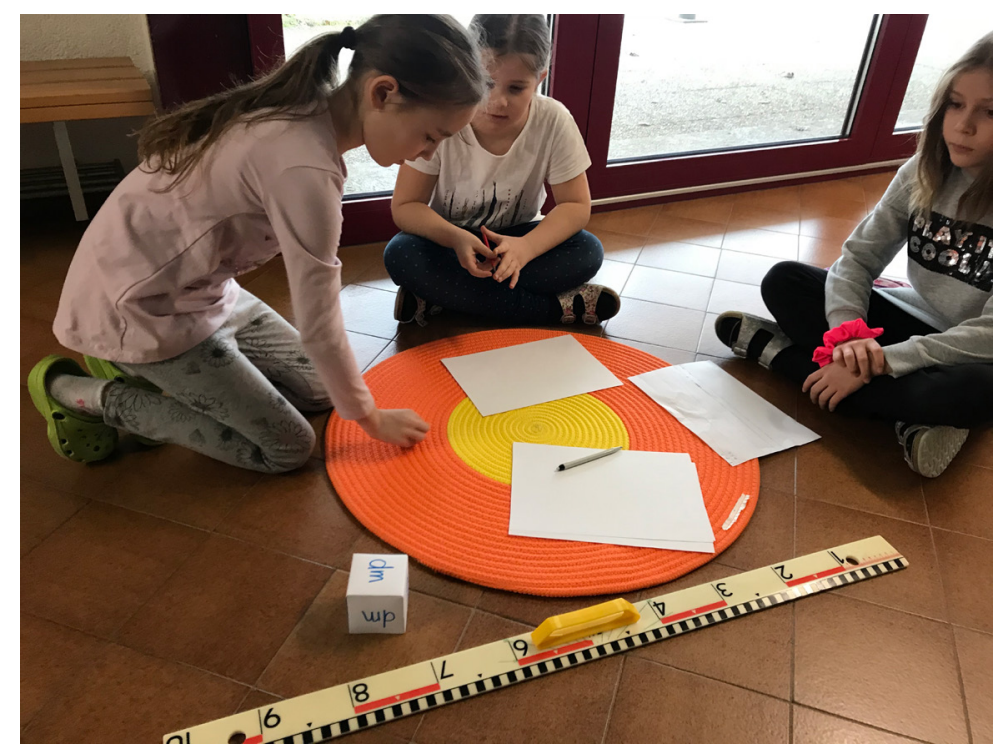

- Misuriamoci: utilizzando il supporto di una scheda (Allegato 7) ciascun bambino completa una sorta di carta d'identità sulle misure del proprio corpo e dell'oggetto preferito. Per rilevare alcune misurazioni è importante che i bambini collaborino con i compagni, utilizzando gli strumenti di misurazione che preferiscono.

- Seguiamo le indicazioni: i bambini scelgono a piacere una tra le diverse attività proposte (Allegato 8), che riguardano la realizzazione di disegni seguendo delle informazioni ben precise. Per riuscire al meglio è indispensabile seguire le indicazioni passo a passo e utilizzare lo strumento di misura (la riga) con attenzione e precisione.

Analogamente a quanto fatto per le misure di tempo, anche per le lunghezze vengono individuate attività significative da svolgere in palestra, durante le ore di educazione fisica.

In questo senso, è interessante la possibilità di far misurare le lunghezze dei diversi campi da gioco, utilizzando strumenti convenzionali o sfruttando i vari attrezzi come unità di riferimento (Figura 11). 
Figura 11

Gli allievi utilizzano le panchine come unità di misura per stabilire la

lunghezza del campo da basket.

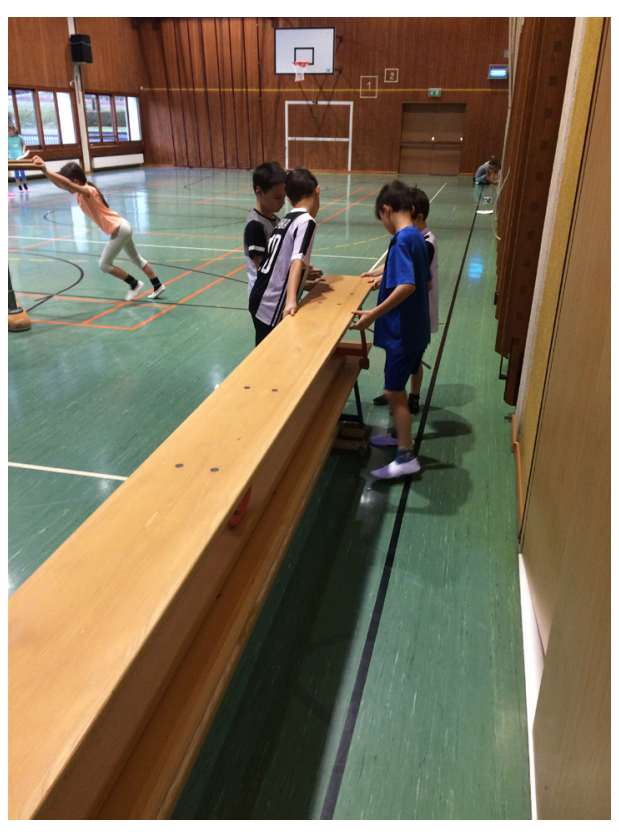

Sono inoltre diversi gli sport in cui le lunghezze sono rilevanti al fine di stabilire il valore di una prestazione: il lancio della pallina o del peso, il salto in lungo, il salto in alto, il salto triplo ecc. Queste discipline possono essere individuate insieme alla classe tramite una discussione. In un secondo tempo, il docente può chiedere agli allievi di praticarle in prima persona, sfruttando le competenze costruite in aula per registrare e confrontare i risultati ottenuti (Figura 12).

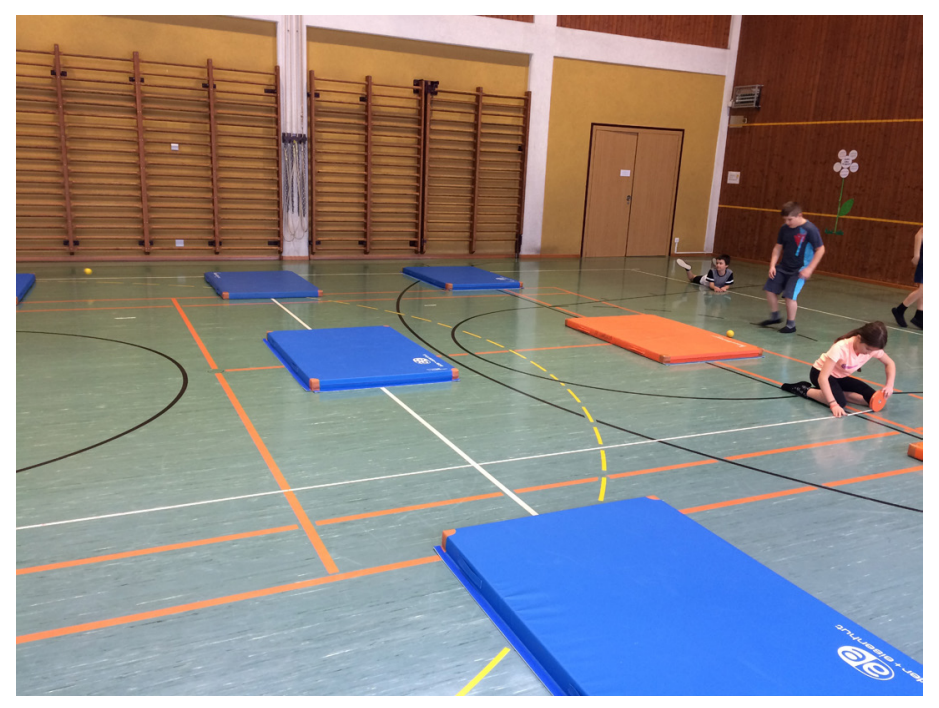

\subsection{Le misure di massa e di capacità e la preparazione di un dolce}

In questa quarta fase del percorso vengono affrontate le ultime due grandezze previste nella progettazione: la massa e la capacità.

L'attività inizia con la proposta, da parte del docente, di realizzare un dolce in vista dei festeggiamenti per la conclusione del progetto, i muffin alle fragole e limone. La cucina è un luogo privilegiato in cui destreggiarsi fra bilance e liquidi da misurare. 
Dopo aver consegnato la scheda con la ricetta (Allegato 9), l'insegnante lascia alla classe il tempo necessario per leggerla e per fare eventuali domande. La maniera in cui sono presentati gli ingredienti, utilizzando cioè varie unità di misura per la massa e per le capacità, dovrebbe far sorgere più di un dubbio: a quanto corrispondono 20 dag di farina? E $10 \mathrm{cL}$ di yogurt? Si propone quindi di mettere da parte la ricetta e di allenarsi per meglio comprendere quel linguaggio, specifico e legato alle due nuove grandezze trattate.

La prima attività di allenamento per queste nuove grandezze prevede che il docente lasci in fondo alla classe una serie di oggetti di diverso tipo: vasi, ciotole, bottiglie ecc., facendo in modo che ci sia almeno un oggetto per ogni allievo (Figura 13). Viene quindi chiesto a ognuno di scegliere l'oggetto che preferisce e di formare una lunga fila, facendo in modo che all'inizio ci sia il bambino con l'oggetto meno capiente, in fondo quello con l'oggetto più capiente. II docente lascia il tempo di discutere, intervenendo solo come mediatore, nel caso in cui gli allievi non riescano da soli a risolvere eventuali disaccordi.

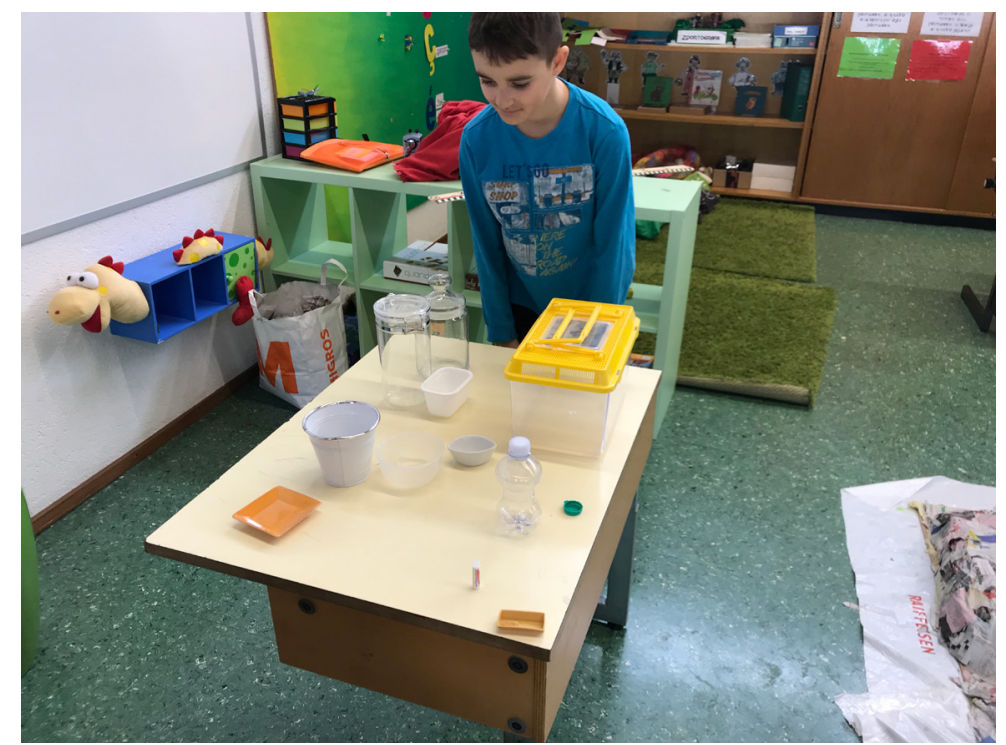

Una volta formata la fila, il docente chiede a ogni allievo di provare a stimare, esprimendosi in maniera libera, la capienza dell'oggetto che ha scelto. Registra le risposte alla lavagna, senza commentare o valutare l'effettiva correttezza delle stesse. II docente invita quindi a osservare e a riflettere sui tipi di unità di misura scelti per stimare la capienza degli oggetti: ce ne sono alcuni simili? Ce ne sono alcuni che sembrano meno adatti o del tutto scorretti? Mediando una discussione, il docente cerca di portare la classe verso la presa di coscienza che, come per le misure di lunghezza, anche per mettersi d'accordo sulla capienza è stato necessario inventarsi un sistema convenzionale.

Con gli stessi oggetti, si ripete l'attività, considerando però la massa e non la capienza: I'ordine sarebbe diverso? Facendo delle analogie con quanto visto in precedenza, e ai cartelloni con la scala delle unità di misure di lunghezza, il docente favorisce la scoperta del grammo (e in un secondo tempo, per analogia, del litro), con le loro sigle e abbreviazioni. Sempre procedendo per analogia, è quindi possibile ricostruire interamente le scale delle unità di misura convenzionali (Figura 14). 
Figura 14

Scala delle unità di misura convenzionali per la massa e per la capacità, appese in classe.

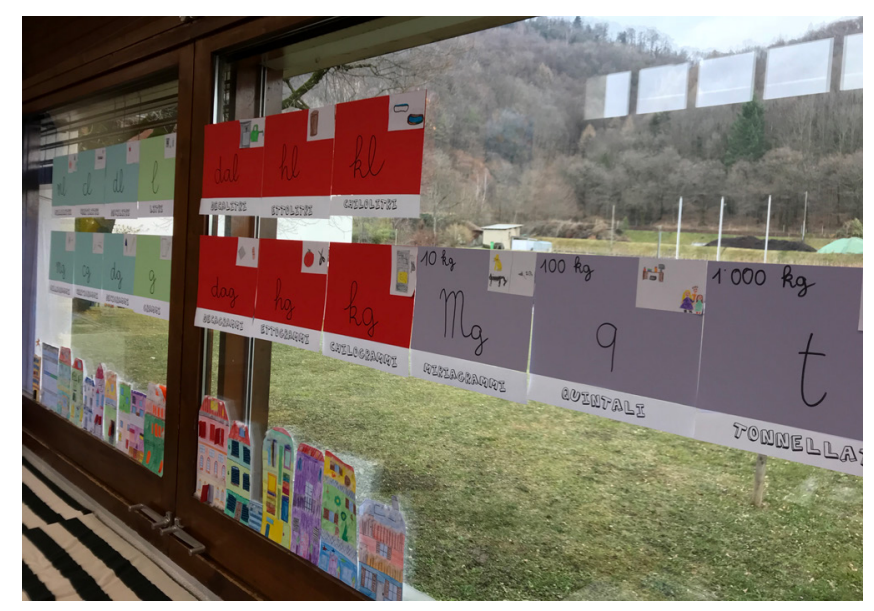

Per facilitare gli allievi, probabilmente meno esperti nel campo delle misure di massa e capacità rispetto a quelle di tempo e lunghezza, può essere utile a questo punto svolgere un'attività che serva a farsi un'idea concreta delle grandezze in gioco; il docente chiede di immaginarsi degli oggetti o dei recipienti che possano rappresentare le unità di misura raccolte nella scala convenzionale: 1 hg può essere il peso ${ }^{4}$ di un mazzo di carte, $1 \mathrm{dL}$ il contenuto di un piccolo bicchiere ecc. Qualora il compito dovesse risultare difficile, il docente e gli allievi possono affidarsi al libro 3 tappi e un barattolo (Julve \& Trius, 2011), su cui si trovano diverse soluzioni originali per ogni unità di misura. In questa fase, è importante far trovare più di una soluzione per ogni unità di misura: 1 kg può essere la massa di una confezione di farina, ma anche del vocabolario a diposizione della classe. Su ogni cartellone con le unità di misura, si mettono almeno due disegni rappresentativi della stessa (Figura 15).

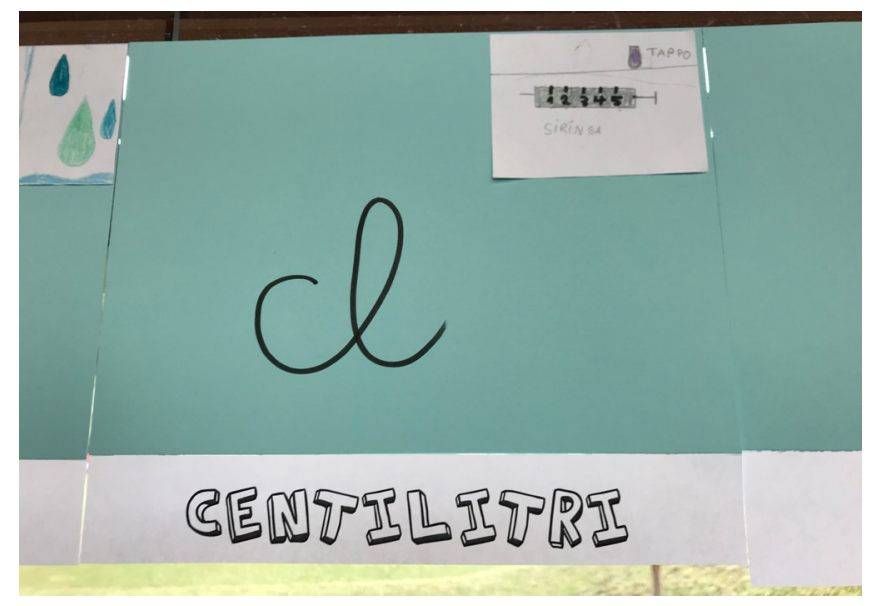

Ora che le unità di misura convenzionali sono definite, è possibile procedere con una serie di attività che permettano di allenarle e di viverle da un punto di vista più pratico. Ecco alcuni esempi:

4. Nonostante la grandezza in gioco sia la massa, con i bambini si è scelto di utilizzare il termine "peso", di uso comune e per loro più familiare. 
1. Misuriamo: la verifica della capacità degli oggetti che in precedenza erano stati presentati per essere riordinati, tramite l'utilizzo di cilindri graduati o di altri strumenti. Allo stesso modo, si verifica anche la massa degli oggetti utilizzando bilance elettroniche $o$ a due piatti.

2. La caccia al recipiente: si parte alla ricerca, in tutta la scuola e a casa, di oggetti che possano contenere dei liquidi. Quindi, si cerca di stabilire quale sia il meno capiente e il più capiente, procedendo prima empiricamente e utilizzando poi strumenti trovati in laboratorio (pipette, cilindri e caraffe graduate ecc.) per verificare le ipotesi.

3. La stima delle misure di massa: attività che segue modalità simili a quelle dei quiz. II docente sceglie un oggetto e lo mostra alla classe. Gli allievi, a gruppetti, discutono e ne ipotizzano la massa, solo tramite l'osservazione. Quindi hanno la possibilità di manipolare e soppesare l'oggetto, e di correggere la propria stima. Si procede alla verifica, e il gruppo che si è maggiormente avvicinato alla massa corretta guadagna un punto. Vince la sfida chi, al termine del quiz, ha guadagnato più punti.

4. La stima delle misure di capacità: attività che avviene in maniera analoga a quella descritta nel punto precedente, ma in cui gli allievi stimano la capienza di alcuni recipienti.

Anche per massa e capacità, le esercitazioni avvengono in parallelo anche in palestra. In particolare, vengono proposte due attività.

Nell'attività della bilancia umana il docente predispone una panchina rovesciata, sopra un coperchio di cassone, a ricreare una grande bilancia a due piatti. I bambini, divisi in due gruppi, hanno a disposizione del materiale di piccole dimensioni e di massa differente. L'attività si svolge sotto forma di staffetta: un bambino deve prendere un oggetto e collocarlo sulla bilancia creata con la panchina. A seguire, gli altri bambini faranno la stessa cosa, collaborando affinché la bilancia risulti in equilibrio al termine del gioco. Lo stesso esercizio può essere in seguito riproposto usando le persone al posto degli oggetti; i bambini hanno l'occasione di confrontarsi e organizzarsi in base alla massa di ognuno, salendo sulla bilancia e facendo in modo che alla fine risulti in equilibrio (Figura 16).

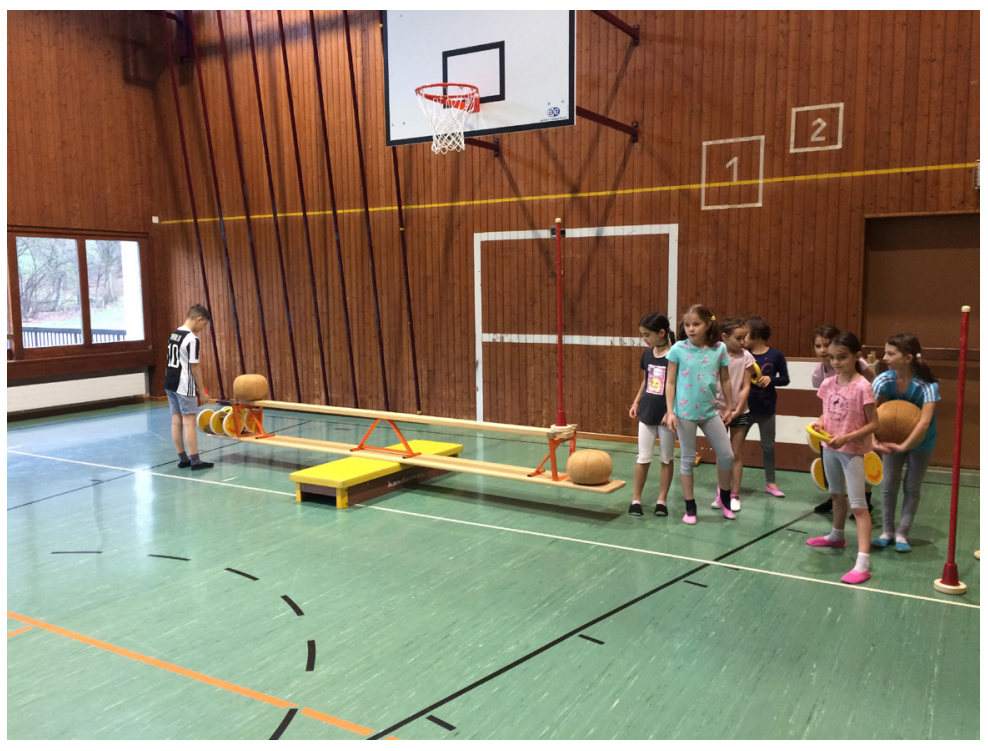


L'attività del trasporto dell'acqua, invece, si svolge fuori dalla palestra. Si tratta di una staffetta competitiva, in cui due o più squadre si sfidano nel trasportare la maggior quantità d'acqua lungo un percorso a ostacoli predisposto dal docente. Per trasportare I'acqua, ognuno può scegliere fra diversi strumenti: spugne, bicchieri bucati, piatti fondi, mestoli. L'acqua va trasportata fino a un catino. Allo scadere del tempo prefissato dal docente, si procede insieme alla misurazione della quantità d'acqua per stabilire il gruppo vincitore. Può essere interessante proporre una discussione sugli strumenti utilizzati per il trasporto: agli allievi si chiede prima di stimare quanto liquido riescono a trasportare, e in seguito si passa alla verifica.

Prima di concludere questa fase, dopo tutti gli allenamenti svolti, il docente propone alla classe di tornare a leggere la ricetta dei muffin. Le unità di misura di massa e capacità, così come l'utilizzo degli strumenti adatti per le misurazioni degli ingredienti, non dovrebbero essere più un problema. La classe viene quindi suddivisa in piccoli gruppi da 3-4 allievi. All'interno dei gruppi vengono suddivisi i compiti, in modo che ognuno debba occuparsi della misurazione di parte degli ingredienti, aggiungendoli di volta in volta al composto. II docente coglie l'occasione per osservare i piccoli cuochi al lavoro, prendendo delle annotazioni relative alle prestazioni degli allievi grazie all'utilizzo di una griglia (per una descrizione più approfondita di questo momento, si faccia riferimento al par. 4).

\subsection{L'ideazione dei giochi per la giornata dei GSFM}

L'invenzione dei giochi avviene nel corso dell'intero anno scolastico, al termine di ognuna delle tre fasi che hanno caratterizzato il lungo percorso, descritte nei par. 3.2, 3.3 e 3.4. Singolarmente o a piccoli gruppi, durante lezioni specificatamente dedicate all'organizzazione dei GSFM, gli allievi propongono dei giochi di movimento legati alle diverse grandezze affrontate in classe $o$ in palestra.

I punti da tenere in considerazione per ideare giochi realizzabili sono stabiliti dal docente:

1. Protagonista del gioco dev'essere una grandezza e la sua relativa misura (misure di tempo, misure di lunghezza, misure di massa e misure di capacità).

2. Ogni gioco deve durare al massimo 30 minuti, considerando anche il momento delle spiegazioni.

3. Il gioco dev'essere una sfida fra due gruppi di circa 10 bambini.

4. Il gioco deve essere adatto ai bambini del primo e del secondo ciclo: in questo senso c'è la possibilità di proporre due varianti, una più semplice e una più complessa.

Le proposte di gioco vengono dapprima presentate alla classe e discusse. In seguito, se non ci dovesse essere una proposta che mette tutti d'accordo, si procede a una votazione per alzata di mano.

Una volta stabiliti i giochi, il docente propone una discussione sul sistema di punteggio: come per i Giochi Senza Frontiere, occorre trovare un modo per tenere i punti, in modo da poter stabilire la squadra vincitrice al termine della giornata. Quando anche questo aspetto è chiarito, all'interno della classe ci si divide i ruoli: gli allievi, divisi in gruppi, sono i responsabili di uno dei quattro giochi e devono occuparsi di scriverne il regolamento e di prepararne il materiale.

In questa fase del percorso, il docente coglie l'occasione per mettere a confronto le 
idee degli allievi mediando discussioni, ponendo domande e cercando di far emergere, in un'ottica costruttiva, le possibili criticità che potrebbero presentarsi nel momento di realizzazione delle sfide. Per facilitare il compito, è auspicabile l'organizzazione di momenti in cui vengono simulate le varie sfide: il gruppo organizzatore gestisce il gioco, un gruppetto partecipa affrontando l'attività e i restanti compagni sono osservatori esterni (Figura 17). Al termine della simulazione, ci si ritrova per uno scambio di opinioni, e per trovare possibili strade per raffinare la proposta in vista dei GSFM veri e propri.

Figura 17

La simulazione di una sfida riguardante le misure di lunghezza.

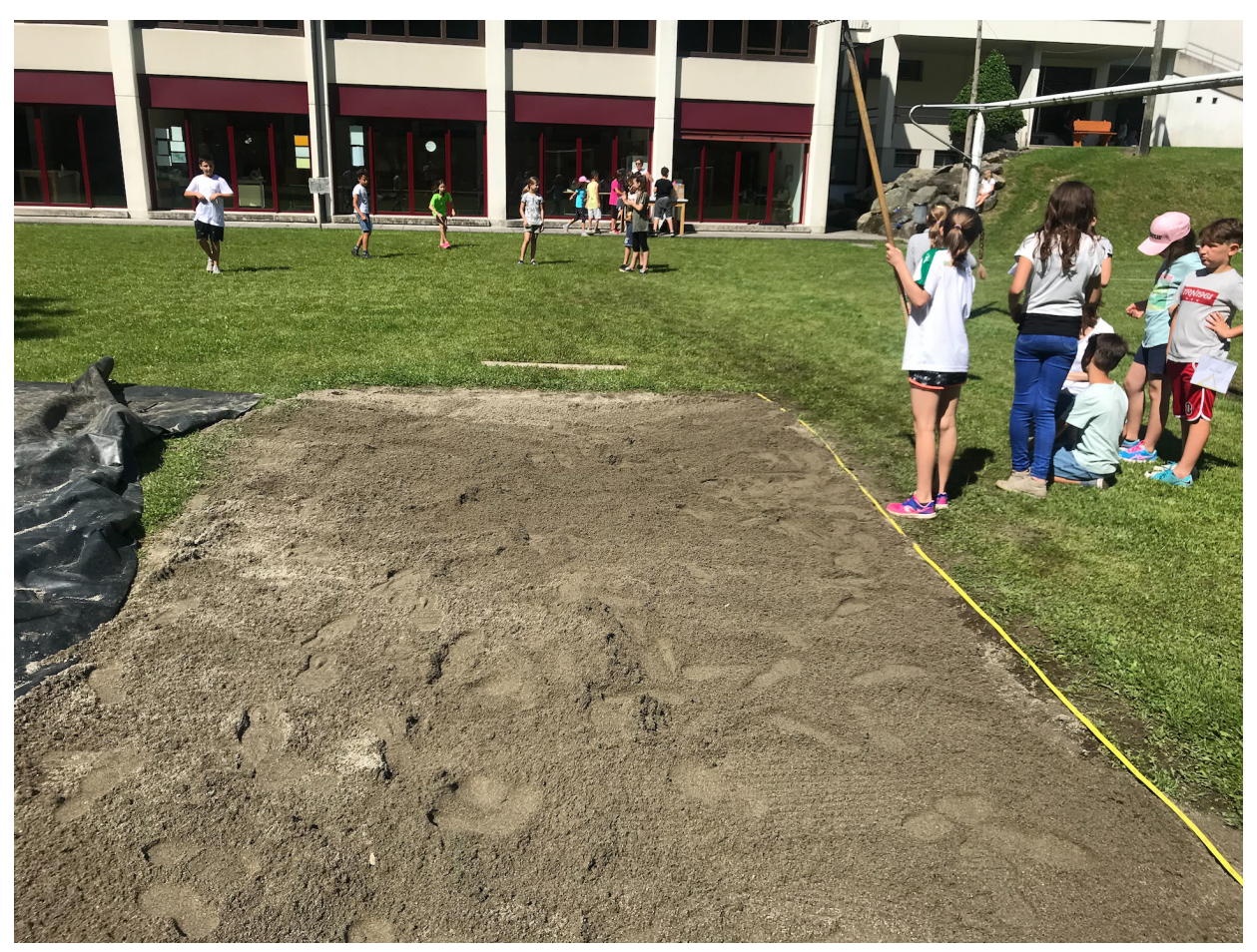

\subsection{La giornata dei GSFM}

La realizzazione ha nella giornata dei giochi il suo momento più forte e significativo. Mettendo a frutto tutte le competenze costruite nel corso dell'anno scolastico, gli allievi spiegano e gestiscono, in collaborazione con i docenti dell'istituto, le sfide legate alle diverse grandezze studiate in classe.

I partecipanti, divisi in gruppi chiamati ognuno con il nome di uno strumento di misura, si sfidano nei quattro giochi preparati dalla classe nel corso dell'intero anno scolastico. A dipendenza dell'andamento delle sfide, guadagnano delle riproduzioni di soldi e monete che potranno impiegare per acquistare bibite e vivande durante il pranzo in comune. Al termine della giornata, dopo un confronto dei risultati ottenuti, vengono proclamati i vincitori, che possono sfilare davanti ai compagni d'istituto come nella migliore tradizione dei Giochi Senza Frontiere.

Il ruolo degli allievi organizzatori è decisamente importante: oltre ad aver preparato il materiale, pianificato la giornata da un punto di vista di spazi e tempi, sperimentato in prima persona i giochi, devono spiegare il regolamento, misurare le prestazioni dei partecipanti, e confrontare i risultati per stabilire i vincitori.

Per farsi un'idea dei giochi progettati e realizzati, e più in generale delle attività proposte durante la giornata dei GSFM, si consiglia la visione di un breve filmato riassuntivo (Allegato 10). 


\section{4 tavaluaraione}

La valutazione delle competenze è un tema senz'altro complesso e delicato, soprattutto in progetti articolati e lunghi come questi. Castoldi (2016) mette in evidenza la necessità di pensare $a$ un impianto valutativo che tenga in considerazione diversi strumenti di analisi e di raccolta dei dati. In questo senso, i momenti di valutazione previsti nel corso del progetto sono stati il più possibile variegati: alcuni avevano lo scopo di spingere l'allievo ad autovalutarsi, altri ancora di favorire la valutazione fra pari, mentre alcuni puntavano a fare un punto su cosa l'allievo sapesse fare, dal punto di vista del docente. Allo stesso tempo, si è cercato di non puntare il riflettore solo sul prodotto, ovvero sul risultato finale del lavoro dell'allievo, ma anche sui processi che hanno portato a tale risultato. Per strutturare gli strumenti di valutazione, è stato necessario un lavoro sugli indicatori di raggiungimento del traguardo, stabilendo a priori quali siano i comportamenti osservabili attesi dall'allievo che indichino il manifestarsi della competenza (Allegato 11).

Gli strumenti di valutazione utilizzati sono riassunti nella Tabella 1.

Tabella 1

Sintesi dei momenti valutativi e descrizione degli strumenti impiegati.

\begin{tabular}{|c|c|}
\hline Chi valuta & Strumento utilizzato \\
\hline Docente & $\begin{array}{l}\text { - Rubrica di prestazione sulla lettura dell'orolo- } \\
\text { gio (utilizzata nella fase del percorso descrit- } \\
\text { ta nel par. 3.2). } \\
\text { - Prova scritta sulle misure di lunghezza } \\
\text { (utilizzata nella fase del percorso descritta } \\
\text { nel par. 3.3). } \\
\text { - Griglia di osservazione sull'utilizzo degli } \\
\text { strumenti per la massa e la capacità (utilizza- } \\
\text { ta nella fase del percorso descritta nel par. 3.4). } \\
\text { - Prova scritta conclusiva sull'intero percorso } \\
\text { annuale legato alle diverse grandezze e } \\
\text { misure (utilizzata al termine del percorso). }\end{array}$ \\
\hline Allievo (autovalutazione) & $\begin{array}{l}\text { - Formulario di autovalutazione sull'intero } \\
\text { progetto e sul percorso annuale dei GSFM } \\
\text { (utilizzato al termine del percorso). }\end{array}$ \\
\hline Compagno (valutazione fra pari) & $\begin{array}{l}\text { - Attività di valutazione fra pari sull'utilizzo de- } \\
\text { gli strumenti di misura della lunghezza (uti- } \\
\text { lizzata nella fase del percorso descritta nel } \\
\text { par. 3.3). }\end{array}$ \\
\hline
\end{tabular}

Di seguito, una descrizione dei diversi momenti valutativi.

\section{Rubrica di prestazione sulla lettura dell'orologio (Allegato 12).}

L'attività di valutazione è svolta individualmente: il docente si occupa di un allievo alla volta, tramite un colloquio, mentre i compagni lavorano autonomamente svolgendo esercizi individuali. Utilizzando del materiale, come gli orologi didattici costruiti e delle immagini relative ad alcuni momenti della giornata (colazione, ricreazione, merenda ecc.), il docente pone delle domande e fa delle richieste all'allievo, registrandone la prestazione in una rubrica. Questo strumento presenta i cinque indicatori di raggiungimento del traguardo nella prima colonna: per ognuno di que- 
sti indicatori sono descritti tre livelli (base, intermedio e avanzato). Al termine del colloquio con l'allievo, il docente fa la somma del punteggio: 0 punti se l'allievo non è stato in grado di rispondere in alcun modo alle richieste avanzate, 1 punto per gli indicatori osservati a livello base, 2 punti per gli indicatori osservati a livello intermedio, 3 punti per gli indicatori osservati a livello avanzato. La prestazione dell'allievo può quindi essere valutata con un punteggio che va da 0 a 15 punti.

Prova scritta sulle misure di lunghezza (Allegato 13).

Si tratta di una prova scritta svolta individualmente, da ogni allievo, e che quindi il docente sottopone alla classe intera allo stesso momento. Le richieste sono facilmente riconducibili agli esercizi e alle attività di allenamento svolte in classe, così come agli indicatori di raggiungimento del traguardo stabiliti per le misure di lunghezza. A ogni esercizio è attribuito un punteggio. Al termine della prova, il docente fa una somma totale dei punti ottenuti da ogni allievo, per un massimo di 12 punti.

Attività di valutazione fra pari sull'utilizzo degli strumenti di misura della lunghezza (Allegato 14).

L'attività viene svolta a coppie, e riguarda la scelta e l'utilizzo di strumenti per misurare delle lunghezze. Un allievo è il misuratore, I'altro è l'osservatore/consigliere. II misuratore ha a disposizione un grande numero di strumenti diversi, e degli oggetti di cui è chiamato a misurare una delle lunghezze. L'osservatore/consigliere ha invece a disposizione un foglio, su cui deve attribuire alla prestazione del compagno un punteggio da 0 a 7 punti, scrivendo eventuali consigli o spunti volti a favorire un miglioramento. II punteggio e i consigli vengono letti dal misuratore e discussi con il consigliere in presenza del docente.

Griglia di osservazione sull'utilizzo degli strumenti per la massa e la capacità (Allegato 15) II docente suddivide la classe in piccoli gruppetti da tre allievi. Ogni gruppo ha il compito di preparare dei muffin alle fragole, misurando gli ingredienti prima di mescolarli fra loro per cuocerli. II momento di misurazione degli ingredienti è svolto individualmente: I'allievo ha a disposizione la ricetta e una moltitudine di strumenti fra cui scegliere. II docente si limita a osservare il suo operato, registrandone in una griglia di osservazione i comportamenti. II doppio + indica una prestazione molto positiva, il + una prestazione generalmente positiva, l'onda una prestazione mediocre, il - una prestazione generalmente negativa, il doppio - una prestazione molto negativa. Per ogni prestazione a cui è stato assegnato almeno un simbolo +, il docente attribuisce quindi un punto, per un totale massimo di 10 punti. Oltre ai simboli, che rendono veloce e immediata la registrazione delle osservazioni, il docente si annota a margine del foglio eventuali comportamenti interessanti o appunti utili.

Formulario di autovalutazione sull'intero progetto e sul percorso annuale dei GSFM (Allegato 16).

Si tratta di un formulario di autovalutazione, in cui l'allievo è chiamato a riflettere sul proprio operato relativo all'intero percorso svolto insieme. Uno dopo l'altro, gli indicatori di raggiungimento del traguardo e i contenuti trattati in classe vengono sottoposti all'allievo, che si trova quindi chiamato a valutare quanto sente di padroneggiare conoscenze, tecniche e processi cognitivi. Oltre a domande legate a contenuti disciplinari, sono previsti interrogativi sull'impegno profuso durante le ore di lavoro in classe. L'allievo è infine chiamato a segnalare se desidera approfondire tematiche ritenute ancora non del tutto chiare. 
Prova scritta conclusiva sull'intero percorso annuale legato alle diverse grandezze e misure (Allegato 17).

Si tratta di una prova scritta svolta individualmente, da ogni allievo, e che quindi il docente sottopone alla classe intera allo stesso momento. Le richieste non sono per forza riconducibili agli esercizi e alle attività di allenamento svolte in classe, ma sono volte a verificare che l'allievo sia in grado di trasferire le proprie competenze affrontando situazioni diverse ma risolvibili grazie alle competenze acquisite. La prova è suddivisa in quattro parti, ognuna legata a una delle diverse grandezze trattate. Gli esercizi sono da svolgere con matita e gomma, scrivendo sul foglio, ma anche utilizzando strumenti e oggetti a disposizione nell'aula. A ogni esercizio è attribuito un punteggio. Al termine della prova, il docente fa una somma totale dei punti ottenuti da ogni allievo, per un massimo di 26 punti.

Tutti i dati raccolti durante le attività di valutazione vanno a confluire, nel corso dell'anno, in una tabella chiamata Rubrica di valutazione (Tabella 2). Si tratta di uno strumento riassuntivo che, analizzato alla fine del percorso, permette di avere una visione sintetica delle competenze e delle prestazioni degli allievi nei diversi momenti dell'anno. Per il docente, la rubrica è uno strumento utile da considerare nel momento in cui è chiamato a esprimere una valutazione numerica legata alla disciplina (per esempio nel compilare la valutazione di fine anno scolastico), ma può anche fungere da spunto per una discussione con l'allievo stesso, in un'ottica formativa.

\begin{tabular}{|c|c|c|c|c|c|c|c|c|}
\hline \multirow[t]{2}{*}{ Allievo } & \multirow{2}{*}{$\begin{array}{l}\text { Tempo } \\
\text { Osservazione da } \\
\text { parte del docen- } \\
\text { te con rubrica } \\
\text { di prestazione } \\
\text { sulla lettura } \\
\text { dell'orologio }\end{array}$} & \multicolumn{2}{|l|}{ Lunghezza } & \multirow{2}{*}{$\begin{array}{l}\text { Massa } \\
\text { e capacità } \\
\text { Osservazione } \\
\text { da parte del } \\
\text { docente sull'uso } \\
\text { dello strumento } \\
\text { durante la pre- } \\
\text { parazione della } \\
\text { merenda }\end{array}$} & \multicolumn{3}{|c|}{ Verifica conclusiva } & \multirow[t]{2}{*}{ Osservazioni } \\
\hline & & $\begin{array}{l}\text { Prova scritta } \\
\text { sulle misure } \\
\text { di lunghezza } \\
\text { (stima e disegno } \\
\text { segmenti) }\end{array}$ & $\begin{array}{l}\text { Valutazione fra } \\
\text { pari sull'uso } \\
\text { degli strumenti } \\
\text { di misura di } \\
\text { lunghezza }\end{array}$ & & $\begin{array}{l}\text { Form } \\
\text { autov } \\
\text { ne su } \\
\text { perco }\end{array}$ & $\begin{array}{l}\text { rio di } \\
\text { Itazio- } \\
\text { tero }\end{array}$ & $\begin{array}{l}\text { Prova scritta } \\
\text { conclusiva } \\
\text { sull'intero } \\
\text { percorso }\end{array}$ & \\
\hline A. & $13 / 15$ & $11 / 12$ & $\begin{array}{l}8 / 10 \rightarrow \\
\text { scegliere stru- } \\
\text { menti adatti }\end{array}$ & $10 / 10$ & $\begin{array}{l}\text { Sì } \\
\text { No } \\
\text { Forse }\end{array}$ & $\begin{array}{l}7 / 11 \\
1 / 11 \\
3 / 11\end{array}$ & $19 / 26$ & $\begin{array}{l}\text { Sbaglia gli orari } \\
\text { mattino e po- } \\
\text { meriggio, qual- } \\
\text { che incertezza } \\
\text { sulle misure di } \\
\text { capacità }\end{array}$ \\
\hline D. & $11 / 15$ & $11.5 / 12$ & $\begin{array}{l}9 / 10 \rightarrow \\
\text { usare strumenti } \\
\text { adatti }\end{array}$ & $\begin{array}{l}4 / 10 \\
\text { Fatica nella } \\
\text { lettura delle } \\
\text { unità di misura }\end{array}$ & $\begin{array}{l}\text { Sì } \\
\text { No } \\
\text { Forse }\end{array}$ & $\begin{array}{l}4 / 11 \\
0 / 11 \\
7 / 11\end{array}$ & $19.5 / 26$ & $\begin{array}{l}\text { Riprendere } \\
\text { la scala delle } \\
\text { misure di massa } \\
\text { e capacità, e } \\
\text { la stima delle } \\
\text { masse }\end{array}$ \\
\hline E. & $14 / 15$ & $10.5 / 12$ & $\begin{array}{l}\text { 9/10 } \rightarrow \\
\text { non distrarsi }\end{array}$ & $8 / 10$ & $\begin{array}{l}\text { Sì } \\
\text { No } \\
\text { Forse }\end{array}$ & $\begin{array}{l}5 / 11 \\
5 / 11 \\
1 / 11\end{array}$ & $23.5 / 26$ & $\begin{array}{l}\text { Imprecisioni } \\
\text { nello scrivere gli } \\
\text { orari mattino/ } \\
\text { pomeriggio }\end{array}$ \\
\hline
\end{tabular}




\begin{tabular}{|c|c|c|c|c|c|c|c|c|}
\hline G. & $15 / 15$ & $10.5 / 12$ & $\begin{array}{l}8.5 / 10 \rightarrow \\
\text { non distrarti! }\end{array}$ & $\begin{array}{l}5 / 10 \\
\text { Fatica nella } \\
\text { lettura delle } \\
\text { unità di misura }\end{array}$ & $\begin{array}{l}\text { Sì } \\
\text { No } \\
\text { Forse }\end{array}$ & $\begin{array}{l}9 / 11 \\
0 / 11 \\
2 / 11\end{array}$ & $23.5 / 26$ & $\begin{array}{l}\text { Difficoltà nelle } \\
\text { misure di massa } \\
\text { e capacità } \\
\text { (stima e utilizzo } \\
\text { delle diverse } \\
\text { unità di misura) }\end{array}$ \\
\hline $\mathrm{H}$. & $14 / 15$ & $9 / 12$ & $10 / 10$ & $\begin{array}{l}6 / 10 \\
\text { Fatica nella } \\
\text { lettura delle } \\
\text { unità di misura }\end{array}$ & $\begin{array}{l}\text { Sì } \\
\text { No } \\
\text { Forse }\end{array}$ & $\begin{array}{l}6 / 11 \\
1 / 11 \\
4 / 11\end{array}$ & $25 / 26$ & $\begin{array}{l}\text { Necessita una } \\
\text { ripresa sulla } \\
\text { scala delle mi- } \\
\text { sure di massa e } \\
\text { capacità }\end{array}$ \\
\hline L. & $15 / 15$ & $11 / 12$ & $10 / 10$ & $\begin{array}{l}6 / 10 \\
\text { Difficoltà uso ci- } \\
\text { lindro graduato }\end{array}$ & $\begin{array}{l}\text { Sì } \\
\text { No } \\
\text { Forse }\end{array}$ & $\begin{array}{l}8 / 11 \\
0 / 11 \\
3 / 11\end{array}$ & 17.5 & $\begin{array}{l}\text { Difficoltà } \\
\text { nell'utilizzo e } \\
\text { nella stima di } \\
\text { misure di massa } \\
\text { e capacità }\end{array}$ \\
\hline M. & $15 / 15$ & $11 / 12$ & $9 / 10$ & $\begin{array}{l}6 / 10 \\
\text { Difficoltà uso ci- } \\
\text { lindro graduato }\end{array}$ & $\begin{array}{l}\text { Sì } \\
\text { No } \\
\text { Forse }\end{array}$ & $\begin{array}{l}8 / 11 \\
1 / 11 \\
2 / 11\end{array}$ & $21.5 / 26$ & \\
\hline P. & $10 / 15$ & $9.5 / 12$ & $\begin{array}{l}5 / 10 \rightarrow \\
\text { scegli strumenti } \\
\text { che conosci! }\end{array}$ & $3 / 10$ & $\begin{array}{l}\text { Sì } \\
\text { No } \\
\text { Forse }\end{array}$ & $\begin{array}{l}5 / 11 \\
3 / 11 \\
3 / 11\end{array}$ & $16 / 26$ & $\begin{array}{l}\text { Insicurezza } \\
\text { generale sulle } \\
\text { ore del mattino/ } \\
\text { pomeriggio, } \\
\text { sulla stima delle } \\
\text { misure di massa }\end{array}$ \\
\hline Q. & $13 / 15$ & $11 / 12$ & $\begin{array}{l}8.5 / 9 \rightarrow \\
\text { scegli strumenti } \\
\text { adeguati }\end{array}$ & $10 / 10$ & $\begin{array}{l}\text { Sì } \\
\text { No } \\
\text { Forse }\end{array}$ & $\begin{array}{l}6 / 11 \\
0 / 11 \\
5 / 11\end{array}$ & $23 / 26$ & \\
\hline
\end{tabular}

Tabella 2

Un esempio di rubrica di

valutazione compilata,

con commenti personali

inseriti dal docente o dai

compagni nelle fasi di

valutazione fra pari. 


\section{Conclusioni e bilancio dell'esperienza}

L'esperienza in aula è stata molto articolata, e di conseguenza fare un bilancio critico completo ed esaustivo è un'operazione delicata e difficile. In questo ultimo paragrafo, ci si limiterà quindi ad esporre, in maniera libera, alcune considerazioni riguardanti potenzialità e criticità delle attività sperimentate.

Percezioni positive sono senz'altro legate all'interdisciplinarità del progetto. La collaborazione con la docente di educazione fisica è stata fruttuosa, e la vicinanza delle tematiche trattate in aula con il tipo di attività proposta in palestra ha permesso una condivisione di senso con la classe particolarmente efficace. La divisione fra aula e palestra è stata comunque piuttosto netta: ai docenti è sembrato interessante che, pur avendo una coerenza di fondo e organizzando momenti di condivisione e di scambio, il docente titolare e la docente specialista mantenessero ruoli distinti, e proponessero delle attività che sentissero proprie, nel rispetto delle diverse aree disciplinari.

La situazione - problema legata all'organizzazione dei GSFM ha funto da motore per l'intero percorso, e ha saputo motivare la classe anche a lungo termine. Dopo il lancio, avvenuto a fine settembre, gli allievi hanno avuto modo di sperimentare, allenarsi e ideare i giochi, realizzando la giornata solo a inizio giugno. Se l'idea di avere una situazione - problema che guidasse l'intera programmazione annuale di matematica per l'ambito Grandezze e misure poneva dei dubbi soprattutto legati a una possibile eccessiva durata nel tempo delle attività, una volta terminata la sperimentazione tutti gli attori coinvolti si sentono di poter consigliare questo tipo di approccio. La motivazione principale, cioè l'organizzazione della giornata dei GSFM, è rimasta sempre viva negli allievi, che hanno potuto così trovare senso nelle diverse fasi del progetto, anche in quelle meno legate allo sport e ai giochi di squadra.

Durante il percorso, a più riprese è stato possibile proporre alla classe situazioni concrete, ancorate al reale. Le attività relative all'ambito Grandezze e misure, in effetti, permettono trasposizioni didattiche particolarmente variegate, legate per esempio alle arti plastiche e alla cucina. Numerose sono state anche le occasioni, nel corso dell'anno, di far effettuare agli allievi misurazioni di ogni tipo in situazioni non previste o programmate. Questa pluralità di sguardi e di situazioni è stata senz'altro vincente, ed è stato naturale tentare di favorirla in ogni modo.

Un ultimo aspetto vincente che si intende evidenziare riguarda la strutturazione della rubrica valutativa, con il tentativo di favorire una varietà di modalità di verifica $\mathrm{e}$ valutazione. Seppure si tratti appunto di un tentativo, e alcuni strumenti utilizzati prestino il fianco a più di una critica da un punto di vista metodologico, l'impressione è che la strada tracciata sia quella giusta. Avere tanti strumenti diversi, e valutare in tanti modi e momenti diversi, fornisce un quadro decisamente completo e variegato dei risultati e delle competenze del singolo allievo. La rubrica, una volta completata, rappresenta un buon riassunto del percorso svolto, e gli elementi in essa registrati sono decisamente più interessanti rispetto a una semplice nota assegnata dopo una verifica di stampo classico, somministrata una volta terminate le attività.

Osservando proprio la rubrica valutativa emerge un primo aspetto negativo. Per motivi di tempo (la parte di percorso sulle misure di lunghezza si è protratta più del previsto) e, forse anche per la natura stessa delle attività proposte, gli allenamenti legati alle misure di massa e di capacità si sono rivelati non del tutto efficaci. Molti allievi hanno riscontrato delle difficoltà nel gestire le scale delle unità di misura convenzionali di queste due grandezze. 
Si può ipotizzare che la decisione di trattarle insieme possa essere non del tutto vincente; probabilmente, concentrarsi su una sola grandezza alla volta avrebbe favorito la comprensione di alcuni concetti e la padronanza delle competenze di base su cui si intendeva lavorare. Le attività stesse, poi, sempre per questioni di tempo sono risultate essere agli occhi degli sperimentatori meno stimolanti ed efficaci rispetto a quelle sulle misure di lunghezza. In futuro, si ritiene importante tentare di concentrarsi di più su esperienze concrete di misurazione, lasciando più tempo per familiarizzare con le unità di misura convenzionali di massa e capacità, di certo meno vicine al vissuto quotidiano del bambino rispetto a quelle di lunghezza o di tempo. Un ultimo aspetto migliorabile riguarda la decisione di proporre un percorso su Grandezze e misure interdisciplinare con l'area motoria, ma in qualche modo slegato dagli altri due ambiti disciplinari previsti per il secondo ciclo (Numeri e calcolo e Geometria), come specificato nel Piano di studio (DECS, 2015). Questa scelta, fatta per evitare che il percorso risultasse ancora più variegato e complesso di quanto non sia già stato, ha però alcuni limiti che è facile immaginare: è impossibile trattare in classe le Grandezze e misure senza che siano implicate anche molte competenze numeriche, e una maggiore attenzione a favorire questo tipo di dialogo intradisciplinare sarebbe auspicabile.

\section{Bibliografia}

Brandenberg, M., Diener, M., von Grünigen Mota Campos, S., Höhtker, B., Keller, B., Keller, R., \& Müller, N. B. (2013). Matematica vol.3. Zürich: Materiale Didattico dei Grigioni.

Castoldi, M. (2011). Progettare per competenze. Roma: Carocci Editore.

Castoldi, M. (2016). Valutare e certificare le competenze. Roma: Carocci Editore.

Cottino, L., Dal Corso, E., Francini, M., Gualandi, C., Nobis, C., Ponti, A., Ricci, M., Sbaragli, S., \& Zola, L. (2011). Misura. Bologna: Pitagora.

Daynes, K. (2016). /l libro dei perché: il tempo. Londra: Usborne Editore.

DECS. (2015). Piano di studio della scuola dell'obbligo ticinese. Disponibile in http://www. pianodistudio.ch/ (consultato il 10.10.2019).

Gottardi, G., \& Gottardi, G. (2016). Imparo con i lapbook - Matematica e scienze - Classe terza. Trento: Erickson Editore.

Julve, Ò., \& Trius, M. (2011). 3 tappi e un barattolo. Roma: Carlo Gallucci Editore.

Novelli, L. (2016). Ciao, sono Tempo. Milano: Francesco Brioschi Editore.

Ungerer, T. (1963). Crictor, il serpente dal cuor d'oro. Bologna: Electa Kids.

\section{Autore/Luca Crivelli}

Dipartimento formazione e apprendimento - SUPSI, Locarno, Svizzera

Icrivelli@supsi.ch 